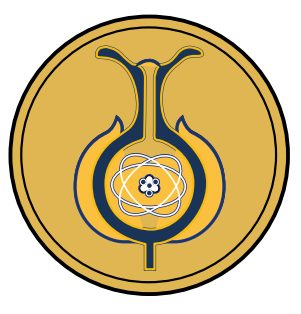

\title{
Guía de análisis de lecciones de libros de texto de Matemáticas en el tema de proporcionalidad
}

\author{
Guidelines for the analysis of mathematics textbooks lessons on the subject of \\ proportionality
}
Guia para a análise de lições de livros didáticos de Matemática sobre o tema da proporcionalidade

\section{María José Castillo Céspedes ${ }^{1}$, María Burgos Navarro ${ }^{2}$, Juan Díaz Godino ${ }^{2}$}

Received: May/25/2021 • Accepted: Set/12/2021 • Published: Jan/31/2022

\section{Resumen (10)}

El objetivo de este trabajo es construir una pauta de indicadores de idoneidad didáctica específicos para el tema de la proporcionalidad, que pueda servir de apoyo para el análisis y valoración de lecciones de libros de texto, y como recurso para la reflexión de los profesores sobre procesos de instrucción efectivamente implementados sobre proporcionalidad. Dado que el estudio del razonamiento proporcional se ha constituido en un campo de investigación relevante en educación matemática, es posible identificar criterios de idoneidad específicos para orientar los procesos de enseñanza y aprendizaje, aplicando la metodología de análisis de contenido a una revisión bibliográfica de investigaciones claves en esta área. Las facetas, componentes e indicadores de la noción de idoneidad didáctica orientan la selección y categorización de conocimientos didáctico-matemáticos sobre la proporcionalidad en educación primaria y secundaria derivados de las investigaciones sobre este contenido. Finalmente, se obtiene una Guía de análisis de lecciones de libros de texto de Matemáticas adaptada al tema de proporcionalidad. Dado que el libro de texto es un material curricular que determina en gran medida lo que sucede en el aula y actúa como mediador en el aprendizaje del estudiante, y que el estudio de las razones, proporciones y la proporcionalidad en los currículos de educación primaria y secundaria es un hecho, la guía obtenida puede ser un recurso valioso para el docente. Es necesario el diseño e implementación de acciones formativas con profesores para su conocimiento y uso competente, entendiendo que no supone una propuesta cerrada.

Palabras clave: idoneidad didáctica; libro de texto; proporcionalidad; formación de profesores

\section{Abstract (1D)}

The objective of this study is the construction of a guideline of indicators of didactic suitability related to the topic of proportionality, which can be used as a basis for the analysis and evaluation of textbook

María José Castillo Céspedes, $\$ mariajosecastilloc.24@gmail.com, (D) https://orcid.org/0000-0002-8046-8927

María Burgos Navarro, $\$ mariaburgos@ugr.es, (D) https://orcid.org/0000-0002-4598-7684

Juan Díaz Godino, \jgodino@ugr.es, (D) https://orcid.org/0000-0001-8409-0258

1 Departamento de Educación Matemática, Facultad de Matemática, Universidad de Costa Rica, San José, Costa Rica.

2 Departamento de Didáctica de la Matemática, Facultad de Ciencias de la Educación, Universidad de Granada, Granada, España. 
lessons, and as a resource for teachers' reflection on effectively implemented instructional processes about proportionality. Since the study of proportional reasoning has become a relevant field of research in mathematics education, it is possible to identify specific suitability criteria for guiding the teaching and learning processes, applying the methodology of content analysis to a bibliographic review of key research in this area. Various aspects, components, and indicators of the notion of didactic suitability guide the selection and categorization of didactic-mathematical knowledge about proportionality in elementary and secondary education derived from research about such content. Finally, a Mathematics Textbook Lesson Analysis Guide adapted to the topic of proportionality was created. Since the textbook contains curricular material that largely determines what happens in the classroom and acts as a mediator in student learning, and ratios, proportions, and proportionality are studied in the curricula of elementary and secondary education, the Guide obtained can be a valuable resource for teachers. It is necessary to design and implement training actions with teachers that they are familiar with and can use competently, bearing in mind that there is always room for further improvement.

Keywords: didactic suitability; textbook; proportionality; teacher education

\section{Resumo}

0 objetivo deste trabalho é construir um padrão de indicadores de idoneidade didática específicos ao tema da proporcionalidade, que possa servir de apoio para a análise e avaliação das aulas de livros didáticos, e como recurso para a reflexão dos professores sobre processos instrucionais efetivamente implementados sobre proporcionalidade. Uma vez que o estudo do raciocínio proporcional se tornou um campo relevante de pesquisa em educação matemática, é possível identificar critérios específicos de aptidão para orientar processos de ensino e aprendizagem, aplicando a metodologia de análise de conteúdo a uma revisão bibliográfica das principais pesquisas nessa área. As facetas, componentes e indicadores da noção de idoneidade didática orientam a seleção e categorização do conhecimento didático-matemático sobre proporcionalidade no ensino fundamental e médio derivado de pesquisas sobre este conteúdo. Por fim, é obtido um Guia para a análise de lições de livros didáticos de Matemática adaptado ao tema da proporcionalidade. Como o livro é um material curricular que determina em grande parte o que acontece em sala de aula e atua como mediador na aprendizagem dos alunos, e que o estudo da razão, das proporções e da proporcionalidade nos currículos do ensino fundamental e médio é um fato, a orientação obtida pode ser um recurso valioso para o professor. É necessário projetar e implementar ações formativas com os professores para seus conhecimentos e uso competente, entendendo que não se trata de uma proposta fechada.

Palavras- chave: idoneidade didática; livro didático; proporcionalidade; formação de professores

\section{Introducción}

El libro de texto continúa siendo el material curricular de uso preferente por parte de profesores y profesoras, determinando en gran medida lo que sucede en el aula y actuando como mediador en el aprendizaje del estudiantado. Esto justifica la importancia que ha adquirido el análisis de los libros de texto como campo de investigación educativa. La cantidad de investigaciones existentes sobre el análisis de libros de texto de Matemáticas da cuenta tanto de su relevancia como de la diversidad de aspectos a contemplar: análisis general en 
relación con sus aspectos formales, motivacionales y metodológicos, estilos de aprendizaje que promueven, análisis de tareas, representaciones, ambigüedades y errores, aspectos afectivos, entre otros (Fan, Zhu y Miao, 2013; Godino, Font y Wilhelmi, 2006; Monterrubio y Ortega, 2011).

La importancia que tiene el libro de texto como recurso principal en la implementación del currículo por parte de la persona docente (AAAS, 2000) nos llevó a elaborar una Guía de análisis de lecciones de libros de texto de Matemáticas (GALT-Matemáticas) (Castillo, Burgos y Godino, 2021), en base a una revisión previa de los antecedentes y teniendo en cuenta las facetas y componentes de la teoría de la idoneidad didáctica (Godino, 2013) para el análisis de un proceso de instrucción matemática. Suponiendo que un profesor o profesora de Matemáticas ha decidido utilizar una lección de un libro de texto como recurso para apoyar el proceso de enseñanza y aprendizaje de algún contenido matemático, la guía de análisis se presenta como una herramienta que le permite valorar la idoneidad didáctica de la lección y apoyar la toma de decisiones fundamentadas sobre su uso en el aula. Sin embargo, como afirman Godino, Rivas y Arteaga (2012),

Queda abierta la necesidad de desarrollar "pautas de análisis y valoración de la idoneidad didáctica" para las distintas áreas de contenido matemático, entendidas como instrumentos que propongan de manera fundamentada indicadores de idoneidad de procesos de aprendizaje y enseñanza de las matemáticas. Es necesario hacer adaptaciones de instrumentos ya existentes para su aplicación en áreas de contenidos particulares, y además "fundamentar" los indicadores de manera explícita, bien en los resultados de investigaciones o bien en juicios expertos asumidos por la comunidad académica, frecuentemente plasmados en las directrices curriculares nacionales e internacionales (Godino et al., 2012, p. 333).

La finalidad de este artículo es construir una Guía de análisis de lecciones de libros de texto de Matemáticas en el tema de proporcionalidad (GALT-proporcionalidad), partiendo de la GALT-Matemáticas anteriormente mencionada y teniendo en cuenta los resultados de investigaciones didácticas sobre la enseñanza y aprendizaje de la proporcionalidad. Esta guía se puede usar también como recurso para orientar la reflexión del profesorado sobre procesos de instrucción efectivamente implementados sobre dicho contenido. Por esta razón incluimos en la guía componentes, como los aprendizajes logrados por la persona estudiante, que tienen más aplicabilidad al análisis de procesos efectivamente implementados, que al caso del análisis de libros de texto.

La elección del tema de la proporcionalidad se debe, en primer lugar, a la importancia que tiene el estudio de las razones, proporciones y la proporcionalidad en los currículos de educación primaria y secundaria, dado su papel transversal respecto a otros temas y sus relaciones con otros contenidos curriculares (Wilhelmi, 2017). Asimismo, el desarrollo del razonamiento proporcional y su relación con el razonamiento algebraico constituyen un campo de investigación muy relevante en educación matemática (Butto y Rojano, 2010; Lesh, Post y Behr, 1988; Lim, 2009) en el que consideramos posible identificar criterios de idoneidad específicos para orientar los procesos de enseñanza y aprendizaje.

El artículo se organiza como sigue: en primer lugar, se describe el marco teórico y la metodología; posteriormente se presenta el 
proceso de elaboración de la guía que contempla una revisión de conocimientos didáctico-matemáticos sobre proporcionalidad orientada a la identificación de criterios de idoneidad específicos de los procesos de enseñanza y aprendizaje; finalmente se incluye una síntesis e implicaciones para el diseño y evaluación de procesos de instrucción.

\section{Marco teórico y metodología}

Entendiendo la lección de un libro de texto como un proceso de instrucción previsto o planificado por el autor del libro, es posible aplicar las herramientas de análisis didáctico del enfoque ontosemiótico (EOS) del conocimiento y la instrucción matemática (Godino, Batanero y Font, 2007) para realizar un análisis sistemático que permita valorar la idoneidad didáctica de dicho proceso, reconocer potenciales conflictos e identificar posibles cambios para mejorar los procesos de enseñanza y aprendizaje en sus diversas dimensiones (Godino, 2013). La teoría de la idoneidad didáctica, como teoría instruccional, junto con sus dimensiones, criterios y desglose operativo, puede servir de guía para la persona docente en las fases de diseño, implementación y evaluación, permitiendo el paso de una didáctica descriptiva-explicativa a una didáctica orientada hacia la intervención efectiva en el aula (Godino, 2013; Godino et al., 2007).

La idoneidad didáctica se entiende como el grado en que un proceso de instrucción reúne ciertas características que permiten calificarlo como óptimo o adecuado, siendo el principal criterio la adaptación entre los significados personales construidos por el alumnado (aprendizaje) y los significados institucionales, ya sean pretendidos o implementados (enseñanza), considerando la influencia del entorno (Godino, 2013).
Esto supone la articulación de criterios relativos a las facetas que intervienen en un proceso de instrucción: epistémica, cognitiva, afectiva, interaccional, mediacional y ecológica (Godino et al., 2007).

Se considera que un proceso de instrucción matemática

tiene mayor idoneidad epistémica en la medida en que los significados institucionales implementados (o pretendidos) representan bien a un significado de referencia. El significado de referencia será relativo al nivel educativo correspondiente y deberá ser elaborado teniendo en cuenta los diversos tipos de problemas y contextos de uso del contenido objeto de enseñanza, así como las prácticas operativas y discursivas requeridas (Godino, 2013, p. 118).

La idoneidad cognitiva concierne al grado en que los significados implementados estén en la zona de desarrollo potencial de las personas estudiantes, así como a la proximidad de los significados personales logrados a los significados pretendidos/implementados. El significado de un objeto matemático refiere a los sistemas de prácticas operativas y discursivas que lleva a cabo una persona (significado personal), o bien que son compartidas en el seno de una institución (significado institucional), para resolver un tipo de situaciones-problema (Godino et al., 2007). Si existe disparidad entre significados de tipo institucional (por ejemplo, entre el significado de referencia y el implementado en un libro de texto) se habla de "conflicto semiótico epistémico", mientras que un desajuste entre el significado manifestado por un sujeto y el de referencia se trata de un "conflicto semiótico cognitivo". 
La idoneidad afectiva alude al grado de implicación (intereses, emociones, actitudes, creencias) del alumnado en el proceso de estudio. Un proceso de instrucción matemática tiene un mayor grado de idoneidad interaccional si las configuraciones didácticas permiten identificar conflictos semióticos potenciales y resolver los conflictos que se producen en el proceso de instrucción. El grado de disponibilidad y adecuación de los recursos materiales y temporales necesarios para el desarrollo óptimo del proceso de enseñanza y aprendizaje determina la menor o mayor idoneidad mediacional. Finalmente, la idoneidad ecológica refiere al grado en que el proceso de instrucción se ajusta al proyecto educativo del centro y al entorno en que se desarrolla.

Las facetas, componentes e indicadores de la teoría de la idoneidad didáctica deben enriquecerse y particularizarse de acuerdo con el tema específico que se quiere enseñar (Breda, Font y Pino-Fan, 2018). Por ello, la valoración de la idoneidad didáctica del proceso de instrucción planificado en una lección de un libro de texto sobre proporcionalidad, así como la identificación de propuestas fundamentadas de su uso en la práctica docente, requiere recopilar, analizar y sistematizar conocimientos didáctico-matemáticos resultado de las investigaciones educativas sobre la enseñanza y aprendizaje de la proporcionalidad.

Por este motivo, se sigue una metodología de análisis de contenido (Cohen, Manion y Morrison, 2011) que permite procesar y revisar dimensiones cualitativas, describir tendencias y especificar características del contenido. Como señalan Godino et al. (2012), esta forma de proceder permite mejorar de manera progresiva los instrumentos de evaluación de la idoneidad didáctica de procesos de instrucción matemática.

En este trabajo, el análisis de contenido se realiza sobre investigaciones claves en relación al análisis de libros de texto y a los aportes teóricos que permitan formular indicadores de idoneidad en cada una de las facetas para el tema de proporcionalidad. Partiendo del trabajo de Aroza, Godino y Beltrán-Pellicer (2016), así como de una revisión y síntesis de otras investigaciones relevantes que aportan conocimientos didáctico-matemáticos sobre proporcionalidad (Fernández y Llinares, 2011; Fiol y Fortuny, 1990; Freudenthal, 1983; Lamon, 2006, 2007; Ruiz y Valdemoros, 2006; Shield y Dole, 2013, entre otras) se adapta el instrumento GALT-Matemáticas a este contenido en concreto.

\section{Elaboración de la Guía de análisis de lecciones de libros de texto de Matemáticas en el tema de proporcionalidad}

En esta sección, se describe la adaptación de la GALT-Matemáticas (Castillo et al., 2021) al tema de proporcionalidad. Prestamos especial atención a las facetas epistémica y cognitiva, ya que son estas donde surgen cambios importantes en cuanto a la adaptación o inclusión de algunos indicadores. No obstante, se incluyen también algunas consideraciones relevantes en el resto de las facetas.

\section{Faceta epistémica}

En la tabla 1 incluimos el conjunto de indicadores para los distintos componentes y subcomponentes de la idoneidad epistémica establecidos en Godino, Burgos, Beltrán-Pellicer, Gea y Giacomone (2019). A continuación, se describen los resultados de las investigaciones que fundamentan la adaptación de indicadores de Godino (2013) o de Aroza et al. (2016), o la inclusión de nuevos indicadores en la guía. 
Tabla 1

Indicadores de idoneidad epistémica en el tema de proporcionalidad

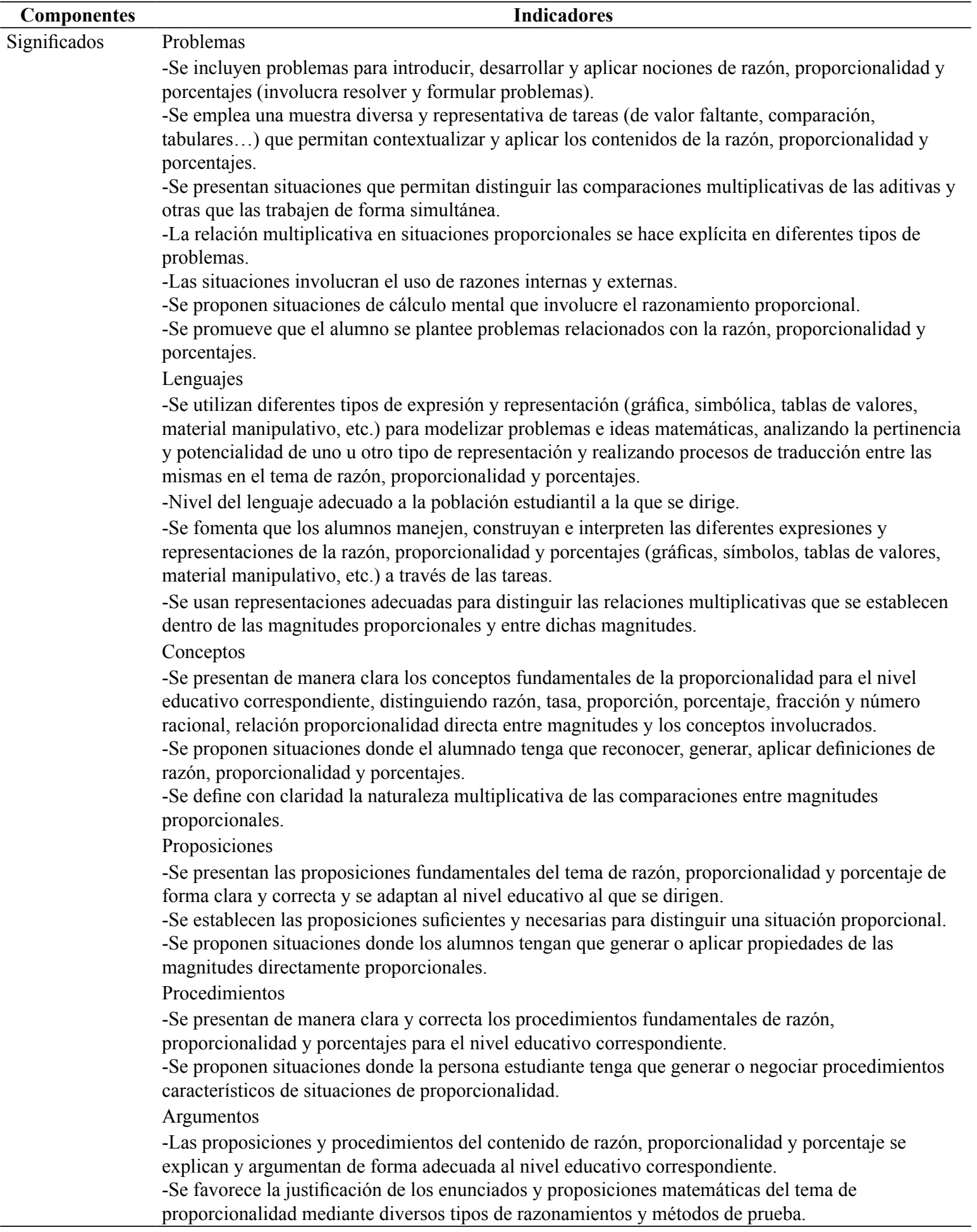




\begin{tabular}{ll}
\hline Componentes & \multicolumn{1}{c}{ Indicadores } \\
\hline Relaciones & -Se establecen relaciones del tema de proporcionalidad con las fracciones y números racionales en \\
& general. \\
& -Se hace explicita la relación entre el bloque aritmético y de magnitudes (se trata la razón entre \\
& números, entre cantidades de una misma magnitud, entre cantidades correspondientes, etc.). \\
& -Se identifican, articulan y desarrollan los cuatro tipos de enfoques o significados de la \\
& proporcionalidad: intuitivo, geométrico, aritmético y algebraico; mediante problemas, \\
& representaciones gráficas, relaciones conceptuales, notaciones matemáticas, procedimientos, etc. \\
& Comunicación, argumentación \\
& -Se promueven diversas situaciones de razón, proporciones y porcentajes donde el alumno tenga que \\
& argumentar y formular conjeturas (describir, explicar, verificar) sobre relaciones de proporcionalidad, \\
& investigarlas y justificarlas. \\
& Modelización \\
& -Se plantean situaciones que permitan al alumno o alumna utilizar el modelo matemático de la \\
& función lineal para representar y comprender relaciones cuantitativas (identificar, seleccionar \\
& características de una situación, representarlas simbólicamente, analizar y razonar el modelo, \\
& las características de la situación, la precisión y limitaciones del modelo) en los temas de razón, \\
& proporcionalidad y porcentajes. \\
& Generalización \\
& -Se proponen situaciones donde el estudiantado tenga oportunidad de describir, explicar y hacer \\
& generalizaciones y conjeturas de patrones geométricos y numéricos, incluso mediante el uso de \\
& expresiones algebraicas (función lineal) relacionados con los temas de razón, proporcionalidad y \\
& porcentajes. \\
& -Los contenidos, situaciones problema y sus soluciones, conceptos, proposiciones, lenguaje etc. \\
& de proporcionalidad y porcentajes se presentan de forma correcta sin errores, contradicciones ni \\
& ambigüedades; se explicitan las condiciones que motivan la condición de proporcionalidad directa, se \\
diferencia una razón de una fracción.
\end{tabular}

\section{Significados}

Problemas. Aroza et al. (2016) distinguen cuatro significados institucionales para la proporcionalidad: intuitivo, geométrico, aritmético y algebraico. Un tratamiento de este tema desde el significado intuitivo-informal supone la presencia de tareas que impliquen la comparación perceptiva de las relaciones proporcionales entre formas geométricas dibujadas a escala (Ruiz y Valdemoros, 2006), o la comparación multiplicativa de los números (Behr, Harel, Post y Lesh, 1992). Por otro lado, desde el enfoque geométrico del tema (semejanza), son representativas las tareas vinculadas a las escalas, que implican realizar ampliaciones y reducciones de figuras que conservan su forma, como las contempladas en Ben-Chain, Keret e Ilany (2012) y Brousseau (1997). Desde el enfoque aritmético de la proporcionalidad, se reconocen esencialmente dos tipos de problemas: de comparación, en los que se dan cuatro valores, relacionados de manera multiplicativa dos a dos, formando dos razones y que se resuelven por un procedimiento netamente aritmético, o de valor faltante (uno de los términos de la proporción es desconocido), que se suelen resolver con la regla de tres (Aroza et al., 2016). Finalmente, situaciones que puedan ser modeladas con una función de la forma $\mathrm{y}=\mathrm{kx}$, en la que $\mathrm{k}$ es la razón constante (constante de proporcionalidad) tienen lugar desde el enfoque algebraico de la proporcionalidad (Aroza et al., 2016).

Con el segundo indicador del subcomponente problemas se valora que la lección del libro de texto incluya una muestra diversa y representativa de tareas en las que se trabajen los distintos enfoques (de valor faltante, comparación, tabulares, etc.). 
Se incluye un tercer indicador en relación a la presencia de situaciones que permitan distinguir las comparaciones multiplicativas de las aditivas y otras que trabajen de forma simultánea. Dado que el razonamiento proporcional supone la capacidad para diferenciar las comparaciones aditivas de las multiplicativas (Behr et al., 1992; Lamon, 2006; Verganud, 1983), investigaciones centradas en el análisis de lecciones de libros de texto en el tema de proporcionalidad (Shield y Dole, 2013) consideran este aspecto como uno de los puntos clave para una instrucción de calidad. Por otro lado, para diversos investigadores, el razonamiento proporcional consiste en reconocer la proporción como una comparación en términos multiplicativos (Cramer y Post, 1993a, 1993b; Lamon, 2007). Por este motivo, el siguiente indicador contempla si se hace explícita la relación multiplicativa en situaciones proporcionales en diferentes tipos de problemas (Shield y Dole, 2013).

Siguiendo las recomendaciones de investigadores como Freudenthal (1983) y Ruiz y Valdemoros (2006), quienes consideran que el pensamiento proporcional cuantitativo implica el manejar indistintamente razones internas y externas (es decir, relaciones establecidas entre diferentes valores de la misma magnitud y entre valores de diferentes magnitudes) al resolver problemas matemáticos, se ha incluido un indicador que permite valorar si las situaciones propuestas por el autor involucran su uso. El penúltimo indicador en este subcomponente, relativo a la presencia de situaciones que involucren el cálculo mental, responde a consideraciones curriculares (MECD, 2014b).

Lenguaje. Además de que se utilicen diferentes tipos de expresión para modelizar problemas, realizando procesos de traducción entre las mismas, que el nivel del lenguaje sea adecuado a quienes se dirige $\mathrm{y}$ que se fomente en el estudiantado utilizar e interpretar las diferentes formas de representaciones de la proporcionalidad y porcentajes, se incluye un cuarto indicador que permite valorar si se usan representaciones adecuadas para distinguir las relaciones multiplicativas que se establecen dentro de las magnitudes proporcionales y entre dichas magnitudes. La importancia de este aspecto, según Shield y Dole (2013), reside en que la estructura de las situaciones proporcionales es una relación entre dos magnitudes y que al considerar esta relación pueden darse los dos tipos de análisis (Vergnaud, 1983).

Conceptos. El primer indicador en este subcomponente contempla la necesidad de presentar de forma clara los conceptos fundamentales de la proporcionalidad, según el nivel educativo; para el caso de secundaria sería necesario distinguir entre razón, tasa, proporción, porcentaje, fracción y número racional, entre otros (Aroza et al., 2016). Este aspecto recibe especial atención en el enfoque aritmético de la proporcionalidad, que tiene un carácter predominante en varias propuestas curriculares e investigaciones (Ben-Chaim et al., 2012; Fernández y Puig, 2002; Hart, 1998; Lamon, 2007; Shield y Dole, 2013). Para Fernández y Puig (2002), el estatuto lógico de la razón es de un nivel más elevado que el de número, fracción, longitud y otros conceptos previos, ya que proviene de una relación de equivalencia. Por otro lado, es importante prestar atención a los conceptos de razón y fracción destacando sus diferencias, ya que la creencia de que estos conceptos son sinónimos puede llevar a potenciales conflictos, frecuentemente identificados en la literatura (Freudenthal, 1983). Al contemplar en el libro de texto este indicador, deben tenerse en 
cuenta tales aspectos, así como distinguir si los conceptos más relevantes se definen de manera explícita y si los términos implicados en su definición también se precisan.

El último indicador de este subcomponente permite valorar si se define con claridad la naturaleza multiplicativa de las comparaciones entre las magnitudes, característica clave de los conceptos involucrados en el tema de proporcionalidad y del razonamiento proporcional (Cramer y Post, 1993a, 1993b; Lamon, 2007).

Proposiciones. En la lección del libro de texto, se deben presentar las proposiciones fundamentales sobre razón, proporcionalidad y porcentajes de manera clara y adecuada, así como aquellas proposiciones suficientes y necesarias para distinguir una situación proporcional: correspondencia biunívoca, monotonía y presencia de una constante de proporcionalidad (Fiol y Fortuny, 1990). Rivas (2013) propone: (a) percatarse de que una razón requiere que exista una relación entre dos cantidades, (b) dicha relación debe ser siempre verdadera en el sentido de que los cambios sobre una de las cantidades implican cambios en la otra cantidad (covariación), (c) dicha covariación debe ser constante: un cambio sobre la primera cantidad implica poder encontrar un cambio sobre la segunda cantidad, y (d) que esa relación entre las cantidades es de tipo multiplicativo. Todas estas consideraciones deben tenerse presentes al valorar el segundo indicador. Además, se considera un tercer indicador para analizar si se proponen situaciones donde las personas alumnas tengan que aplicar propiedades de las magnitudes directamente proporcionales.

Procedimientos. En este subcomponente se valora que se presenten de manera clara y correcta los procedimientos fundamentales en relación a razón, proporcionalidad (obtención de la constante de proporcionalidad, reducción a la unidad, planteamiento y resolución de la ecuación proporcional, definición y manipulación de la función lineal) y porcentajes. Es importante también que se incluyan situaciones donde cada alumno y alumna tengan que generar o negociar procedimientos característicos de situaciones de proporcionalidad.

Argumentos. En este subcomponente se consideran dos indicadores: que las proposiciones y procedimientos en relación a la razón, proporcionalidad y porcentaje se expliquen de forma adecuada al nivel educativo correspondiente; que se favorezca la justificación de los enunciados y proposiciones mediante diversos tipos de razonamientos y métodos de prueba. A este respecto, mencionemos que para Lamon (2007) "el razonamiento proporcional significa aportar razones que sustenten afirmaciones hechas sobre las relaciones estructurales entre cuatro cantidades (a, b, c, d) en un contexto que simultáneamente involucre la covarianza de cantidades y la invariancia de razones o productos" (p. 637).

\section{Relaciones}

Existen conexiones imprescindibles que deben trabajarse en el tema de proporcionalidad: por un lado, la relación con el tema de fracciones y números racionales (Freudenthal, 1983; MECD, 2014b; Shield y Dole, 2013); por otro, el vínculo entre el bloque aritmético y de magnitudes, conexión que muchas veces no se hace explícita en el tratamiento del tema en los libros de texto debido a que se considera la razón como un número únicamente (Gairín y Oller, 2012; Guacaneme, 2001).

El último indicador en esta componente hace hincapié en la interrelación entre los diversos significados de la proporcionalidad 
que construyen el significado global para este contenido. El significado global, que se sigue tanto de los contextos de uso intra-matemáticos (aritmético, algebraico-funcional, geométrico, probabilístico, estadístico) como extra-matemáticos (ámbitos científico-técnico y artístico, vida cotidiana) debe permitir un tránsito flexible entre los distintos significados parciales (Wilhelmi, 2017). Además, el estudio del tema de proporcionalidad basado exclusivamente en uno de estos enfoques puede generar obstáculos epistemológicos (Aroza et al., 2016); de ahí la importancia de contemplar relaciones entre estos.

\section{Procesos matemáticos}

Los conceptos de razón, proporción y proporcionalidad adquieren un significado unificado con la noción de función lineal, que puede "considerarse como la matematización de las nociones cotidianas y utilitarias de la proporcionalidad" (Fiol y Fortuny, 1990 , p. 83). Por este motivo se ha precisado, en el indicador referido al proceso de modelización, valorar si se promueve que el alumno utilice el modelo matemático de la función lineal. En este sentido, autores como Bolea, Bosch y Gascón (2001) y Obando, Vasco y Arboleda (2014) describen el razonamiento proporcional como aquel que comprende una función lineal en un sistema de dos variables y permite extraer conclusiones de una situación que puede caracterizarse por una razón constante entre cantidades de magnitudes.

Partiendo de que la generalización constituye un rasgo característico del pensamiento algebraico, y la consideración del razonamiento proporcional como ruta de acceso al pensamiento algebraico (Butto y Rojano, 2010; Lim, 2009), se incluye un indicador para valorar si la lección promueve situaciones donde los estudiantes tengan la oportunidad de describir, explicar y hacer generalizaciones y conjeturas sobre patrones numéricos y geométricos que permitan avanzar desde el tratamiento aritmético de la razón y proporción hasta el algebraico basado en la función lineal.

\section{Conflictos epistémicos}

En este componente se tiene en cuenta la existencia de acciones que constituyen potenciales obstáculos epistemológicos para el desarrollo del razonamiento proporcional. Una de ellas consiste en obviar las condiciones necesarias y suficientes para determinar si una relación es o no de proporcionalidad directa (Bosch, 1994; Fiol y Fortuny, 1990; Rivas, 2013). Además, el uso de situaciones pseudo-proporcionales, referidas a problemas cuya estructura lingüística es similar a uno de proporcionalidad (Modestou, Elia, Gagatsis, y Spanoudis, 2008), puede generar conflictos al asumirlos como proporcionales. Así, un error comúnmente reconocido consiste en la "ilusión de la linealidad", esto es, aplicar procedimientos lineales a la resolución de problemas no lineales (Van Dooren, De Bock, Depaepe, Janssens y Verschaffel, 2003).

\section{Faceta cognitiva}

En la tabla 2 incluimos el conjunto de indicadores de esta faceta para los componentes y subcomponentes establecidos en Godino et al. (2019). A continuación, hacemos referencia a los resultados de investigaciones que fundamentan el ajuste o incorporación de nuevos indicadores respecto a Godino (2013) o Aroza et al. (2016). 
Tabla 2

Indicadores de idoneidad cognitiva en el tema de proporcionalidad

\begin{tabular}{|c|c|}
\hline Componentes & Indicadores \\
\hline \multirow{4}{*}{$\begin{array}{l}\text { Significados } \\
\text { personales } \\
\text { (Aprendizaje) }\end{array}$} & Comprensión \\
\hline & $\begin{array}{l}\text {-Las experiencias (situaciones, ejemplos, explicaciones...) propuestas permiten valorar si el } \\
\text { alumno o la alumna comprende los conceptos, procedimientos, proposiciones y las mismas } \\
\text { situaciones en el tema de razón, proporcionalidad y porcentajes. Importa especialmente que se } \\
\text { promueva la comprensión de: conceptos de covariación, invariancia, comparación, los cambios } \\
\text { absolutos y relativos, el sentido de razón, y las relaciones matemáticas involucradas en situaciones } \\
\text { proporcionales. }\end{array}$ \\
\hline & Competencia \\
\hline & $\begin{array}{l}\text {-Las experiencias (situaciones, ejemplos, explicaciones...) propuestas permiten valorar si cada } \\
\text { estudiante desarrolla la competencia comunicativa, argumentativa y fluencia procedimental. } \\
\text { Interesa especialmente que sea capaz de: usar razones y proporciones, manejar las razones } \\
\text { internas y externas, distinguir situaciones proporcionales y no proporcionales, resolver situaciones } \\
\text { multiplicativas y aditivas. }\end{array}$ \\
\hline Relaciones & $\begin{array}{l}\text {-Las experiencias (situaciones, ejemplos, explicaciones...) propuestas permiten valorar si } \\
\text { el estudiantado establece relaciones o conexiones entre los objetos matemáticos y entre sus } \\
\text { correspondientes significados: intuitivo, geométrico, aritmético o algebraico en diferentes contextos. }\end{array}$ \\
\hline $\begin{array}{l}\text { Conocimientos } \\
\text { previos }\end{array}$ & $\begin{array}{l}\text {-Se contemplan los conocimientos previos necesarios de acuerdo al nivel educativo correspondiente. } \\
\text {-Los contenidos pretendidos se pueden alcanzar (tienen una dificultad manejable) en sus diversas } \\
\text { componentes. }\end{array}$ \\
\hline Diferencias & -Se incluyen actividades de ampliación y de refuerzo. \\
\hline individuales & $\begin{array}{l}\text {-Se promueve el acceso, el logro y apoyo de todo el estudiantado, por ejemplo, promoviendo uso de } \\
\text { diversas estrategias correctas (multiplicativas, de construcción progresiva, razón unitaria, factor de } \\
\text { cambio o factor escalar, comparación de razones, algoritmo del producto cruzado o regla de tres, etc.). }\end{array}$ \\
\hline \multirow[t]{3}{*}{$\begin{array}{l}\text { Conflictos } \\
\text { cognitivos }\end{array}$} & $\begin{array}{l}\text {-Se valora y emplea el uso de estrategias erróneas (estrategias aditivas...) como fuente de } \\
\text { aprendizaje. }\end{array}$ \\
\hline & $\begin{array}{l}\text {-Se prevén situaciones con diferentes niveles de dificultad (que involucren números enteros, no } \\
\text { enteros, relaciones de divisibilidad entre cantidades, que se altere el orden de los datos en los } \\
\text { problemas...). }\end{array}$ \\
\hline & $\begin{array}{l}\text {-Se advierte de errores y dificultades del alumnado tanto conceptuales como procedimentales } \\
\text { (obstáculo de linealidad, asumir como suficientes condiciones necesarias (covariación), ignorar parte } \\
\text { del problema, realizar operaciones al azar, usar estrategias aditivas erróneas...). }\end{array}$ \\
\hline \multirow[t]{5}{*}{ Evaluación } & -Se proponen instrumentos de evaluación o autoevaluación. \\
\hline & -Se promueve que los resultados de las evaluaciones se difundan y usen para tomar decisiones. \\
\hline & -Los diversos modos de evaluación son adecuados para evaluar que los alumnos y alumnas logran \\
\hline & $\begin{array}{l}\text { la apropiacion de los conocimientos y competencias pretendidas (comprension conceptual y } \\
\text { proposicional; competencia comunicativa y argumentativa; fluencia procedimental; comprensión }\end{array}$ \\
\hline & $\begin{array}{l}\text { situacional; competencia de modelización y generalización, competencia metacognitiva). } \\
\text {-La evaluación tiene en cuenta distintos niveles de comprensión y competencia. }\end{array}$ \\
\hline
\end{tabular}

En el caso del análisis de una lección del libro de texto, puede ser más complicado o incluso menos pertinente aplicar los componentes de significados personales, relaciones y procesos matemáticos, ya que hacen referencia a aprendizajes de sujetos individuales. Se pueden identificar, no obstante, algunos conflictos cognitivos potenciales relacionados con los conocimientos previos requeridos. También es posible observar si el autor de la lección tiene en cuenta posibles adaptaciones a diferencias individuales y propone actividades de evaluación.

\section{Significados personales}

Comprensión. En este subcomponente se valora si el proceso instruccional promueve la comprensión de los conceptos de covariación, invariancia, comparación, así como de los cambios absolutos y relativos, 
el sentido de razón y las relaciones matemáticas involucradas en situaciones proporcionales. Las nociones de comparación y covariación (relación existente entre dos magnitudes, de manera que todo aumento o disminución de una de ellas se traduce en un aumento o disminución de la otra) constituyen la base del razonamiento proporcional y son los soportes conceptuales de la razón y la proporción (Lesh et al., 1988). Aspectos como los cambios absolutos y relativos (aditivos/multiplicativos), el sentido de razón (relación multiplicativa) e invariancia (entre razones) están fuertemente relacionados con la adquisición del razonamiento proporcional (Cramer y Post, 1993a, 1993b; Lamon, 2007).

Competencia. El razonamiento proporcional implica ser capaz de diferenciar las comparaciones aditivas de las multiplicativas (Behr et al. 1992; Lamon, 2006; Shield y Dole, 2013; Verganud, 1983). Por otro lado, para investigadores como Freudenthal (1983) o Ruiz y Valdemoros (2006), el pensamiento proporcional cuantitativo se adquiere cuando se es capaz de usar las razones y proporciones y utilizar indistintamente razones internas y externas. Por este motivo, en este subcomponente se considera si la lección permite contemplar si el alumno o la alumna es capaz de: usar razones y proporciones, manejar las razones internas y externas, distinguir situaciones proporcionales y no proporcionales y resolver situaciones multiplicativas y aditivas.

\section{Relaciones}

En este componente se analiza si las situaciones propuestas permiten valorar si el alumnado establece conexiones entre los diferentes objetos matemáticos (razón, proporción, relación de proporcionalidad, función lineal, etc.) y entre sus diversos significados.

\section{Conocimientos previos}

En este componente se valora si se contemplan los conceptos (fracciones, equivalencia de fracciones, números decimales, magnitudes, entre otras) y las habilidades que son requisitos previos para que las personas estudiantes establezcan las conexiones adecuadas entre los distintos objetos matemáticos. Se analiza también si los conceptos, procedimientos y proposiciones en relación con la proporcionalidad se presentan en un grado creciente de complejidad (Monterrubio y Ortega, 2012).

\section{Diferencias individuales}

En la lección se debe garantizar que todo el estudiantado logre un adecuado razonamiento proporcional. En particular, esto requiere la capacidad de desarrollar múltiples estrategias (multiplicativas, de construcción progresiva, la razón unitaria, factor de cambio o factor escalar, comparación de razones, algoritmo del producto cruzado o regla de tres) para resolver diversos tipos de situaciones problema (Cramer y Post, 1993b; Lesh et al., 1988; Silvestre y Da-Ponte, 2011; Tourniaire y Pulos, 1985).

\section{Conflictos cognitivos}

Van Dooren et al. (2003) afirman que a menudo las personas estudiantes utilizan un razonamiento proporcional en circunstancias que no lo requieren (por ejemplo, situaciones constantes, no lineales y aditivas), y este es uno de los errores más dominantes. De forma similar se describe la tendencia a abordar situaciones proporcionales de forma aditiva en lugar de multiplicativa (Cramer y Post, 1993b; Lamon, 1993; Tourniaire y Pulos, 1985), evitando el uso de fracciones (Hart, 1988). Estas estrategias erróneas deben ser valoradas y empleadas como fuente de aprendizaje. 
Por otro lado, es importante valorar el nivel de dificultad de las tareas en el tema de proporcionalidad. Al respecto, varias investigaciones (Fernández y Llinares, 2011; Lamon, 1993; Karplus, Pulos y Stage, 1983; Turnaire y Pulos, 1985; Van Dooren, De Bock, Gillard y Verschaffel, 2009) evidencian la existencia de diversos factores que intervienen en el nivel de dificultad de las tareas de proporcionalidad, entre ellos: la relación entre los números involucrados, el uso de razones enteras y no enteras, las unidades de las magnitudes que aparecen en la situación, el formato en que se presenta la tarea, la familiaridad con el contenido, el orden en que se presentan los datos en problemas de valor faltante, entre otros. Finalmente, es importante que la lección del libro de texto advierta al alumnado de estos posibles errores.

\section{Evaluación}

En este componente se valora si el libro de texto incluye diversos instrumentos de evaluación, coevaluación y autoevaluación que se apliquen en contextos variados. Los diversos modos de evaluación deben permitir apreciar el grado de adquisición de los conocimientos y competencias pretendidas. Además de la comprensión conceptual y proposicional, la competencia comunicativa y argumentativa o la flexibilidad procedimental, adquiere importancia la evaluación de la competencia de modelización y generalización que no aparecía considerada en Godino (2013) o Aroza et al. (2016). Además, la importancia de la metacognición (NCTM, 2000) lleva a valorar la existencia de preguntas o situaciones que permitan evaluar la habilidad reflexiva de cada estudiante para tomar conciencia de lo que hacen, autoevaluar sus progresos y ajustar sus estrategias en la resolución de problemas.

\section{Faceta afectiva}

A pesar de que no existe un consenso para delimitar los aspectos que se deben considerar al definir el dominio afectivo, la mayoría de los autores y autoras incluyen las emociones, actitudes, creencias y valores (Beltrán-Pellicer y Godino, 2019). En este caso, la consideración del contenido específico no supone cambios relevantes en los indicadores que se consideran para cada una de estas componentes con respecto a la GALT-Matemáticas (Castillo et al., 2021). Sin embargo, mencionamos aquellos aspectos de las investigaciones previas sobre el dominio afectivo que tienen que ver con la proporcionalidad.

El aula debe ser un espacio social que fomente la participación, la responsabilidad en el trabajo, el interés y la motivación por la materia (Aroza et al., 2016). En el tema de proporcionalidad, existen evidencias de que la persona estudiante suele tener un mejor desempeño cuando participa de actividades colaborativas (Ben-Chaim, Fey, Fitzgerald, Benedetto y Miller, 1998; Burgos y Godino, 2018); además la participación, el aprendizaje en contexto, así como el uso de juegos interactivos permiten mejorar las habilidades del razonamiento proporcional (Martínez, 2015; Vandercruysse et al., 2016). Por otro lado, investigaciones experimentales respaldan que la resolución de problemas sobre proporcionalidad utilizando materiales de robótica fomentan también la motivación, el interés, las buenas actitudes y emociones de alumnos y alumnas (Martínez, 2015). Así, la idoneidad afectiva del proceso instruccional planificado en una lección de libro de texto se puede incrementar incluyendo tareas de trabajo colaborativo o incorporando materiales y recursos como los mencionados. 


\section{Faceta instruccional (interac- cional y mediacional)}

Como ocurría en la faceta afectiva, la consideración del contenido específico no supone cambios relevantes en los indicadores respecto a la GALT-Matemáticas (Castillo et al., 2021). Precisamos a continuación aquellos aspectos que sí son particulares del tema de la proporcionalidad.

Existe un amplio consenso en la comunidad científica sobre la pertinencia de iniciar el estudio de la proporcionalidad con experiencias intuitivas correspondientes a una aproximación cualitativa de la proporcionalidad, basada en actividades de estimación (Fernández, 2001; Fiol y Fortuny, 1990; Piaget, 1978). Se propone una secuencia didáctica que permita avanzar desde un conocimiento de naturaleza cualitativa de estructura aditiva (pre-proporcional) hacia un conocimiento cuantitativo de estructura multiplicativa, haciendo uso de procesos que fomenten la manifestación de estrategias de construcción progresiva que permitan una consolidación gradual del razonamiento proporcional (Cramer y Post, 1993a; Ruiz y Valdemoros, 2006). En este sentido, conviene retrasar la introducción de la regla de tres hasta que las personas aprendices hayan adquirido suficiente experiencia en el tema (Cramer, Post y Currier, 1992; Monchón, 2012; Obando et al., 2014; Shield y Dole, 2013; Tourniaire y Pulos, 1985).

En el aspecto mediacional se consideran dos componentes: recursos materiales y tiempo. En relación con el primero, la lección del libro de texto debe promover la incorporación de materiales complementarios al propio como: computadora, escalímetro, pantógrafo, compás de proporción, entre otros para trabajar el tema (Grupo Beta, 1990).

\section{Faceta ecológica}

La faceta ecológica contempla en primer lugar el grado en que los contenidos, objetivos y situaciones se adecuan a las directrices curriculares (AAAS, 2000). Además, estos contenidos deben contribuir activamente a la formación socioprofesional de la persona estudiante, estableciéndose relaciones intra e interdisciplinares (Castillo et al., 2021).

En el currículo español el tema de proporcionalidad tiene una presencia longitudinal y transversal, apareciendo vinculada a materias como Educación Artística, Física, Química, Biología, Dibujo Técnico, Educación Plástica, Visual y Audiovisual, entre otras (Wilhelmi, 2007). El tema aparece en primaria vinculado al bloque denominado "Números" (MECD, 2014a). En dicho bloque se trata principalmente los conceptos de: razón y proporción, magnitudes directa e inversamente proporcionales, constante de proporcionalidad, porcentajes, así como las leyes del doble, triple, mitad y aplicaciones en la vida cotidiana. Por su parte, en secundaria la proporcionalidad se encuentra en los bloques denominados "Números y Álgebra", "Geometría", "Funciones" y "Estadística y Probabilidad" (MECD, 2014b). En el bloque de Números y Álgebra se incluyen los contenidos de: porcentajes (aumentos y disminuciones), la razón y proporción, magnitudes directa e inversamente proporcionales, la constante de proporcionalidad, resolución de problemas, y repartos directa e inversamente proporcionales. Desde el punto de vista geométrico, la proporcionalidad aparece relacionada a los contenidos de semejanza y el teorema de Tales. En el bloque de Funciones se trabaja desde la función lineal, específicamente en representaciones gráficas de modelos, incluyendo el caso de proporcionalidad inversa. Por último, el bloque de 
Estadística y Probabilidad se relaciona con los contenidos de: media, desviación típica de la media muestral y proporción muestral, e intervalos de confianza.

La formación socioprofesional supone, en primer lugar, que los alumnos y alumnas tengan oportunidades de desarrollar el razonamiento proporcional en su vida diaria (Karplus et al. 1983; Lamon, 1993), para lo que es fundamental que se incluyan situaciones contextualizadas en la lección del libro de texto. Además, diferentes estudios dan cuenta de la utilidad de la proporcionalidad en trabajos u oficios variados (agricultura, construcción, pintura, marmolería, albañilería) y en el contexto comercial que implica el manejo de dinero, pago o cobro de servicios, de mercancías, entre otros (Ávila, 2013; Carraher, Carraher y Schliemann, 1991; Soto y Rouche, 1995).

\section{Síntesis e implicaciones}

Como señalan Breda et al. (2018), los criterios de idoneidad, entendidos como normas de corrección que establecen cómo debería realizarse un proceso de enseñanza y aprendizaje, deben ser consensuados por la comunidad interesada en la educación matemática, o por un sector relevante de esta, dado que orientan cómo se deberían hacer las cosas y además permiten valorar el proceso de enseñanza y aprendizaje previsto o implementado.

En este trabajo hemos abordado el problema de identificar, en las investigaciones didácticas sobre enseñanza y aprendizaje de la proporcionalidad en educación primaria y secundaria, resultados que se pueden interpretar como indicadores de idoneidad didáctica de los procesos de instrucción sobre dicho contenido matemático. Partiendo de la revisión de la literatura específica y apoyándonos en las facetas y componentes de la teoría de la idoneidad didáctica para categorizar los indicadores relativos a las distintas facetas, hemos construido el instrumento GALT-proporcionalidad, concretando cambios relevantes en las tablas 1 y 2 . En el caso de las dimensiones epistémica y cognitiva, ha sido especialmente necesario ampliar los criterios generales de idoneidad propuestos en trabajos previos (Aroza et al., 2016; Castillo et al., 2021; Godino, 2013) con indicadores específicos del contenido matemático, en nuestro caso la proporcionalidad.

Dado el uso intensivo que se hace del libro de texto en la práctica docente, la guía de análisis aquí presentada puede ser un recurso valioso para la persona docente; no obstante, es necesario diseñar e implementar acciones formativas con el profesorado para su conocimiento y uso competente.

El sistema de componentes, subcomponentes e indicadores de la idoneidad didáctica en sus distintas facetas usado en este trabajo para el tema de proporcionalidad no es una propuesta cerrada. Como síntesis de conocimientos didáctico-matemáticos a tener en cuenta en el diseño, implementación y evaluación de procesos de instrucción matemática, se trata de una herramienta cuya aplicación y discusión, tanto por los investigadores como por los propios profesores y profesoras, permitirá su progresiva mejora $\mathrm{y}$ enriquecimiento. Constituye un puente entre el complejo entramado de información didáctica disponible (contenida en trabajos de investigación, documentos curriculares, recursos instruccionales, entre otras fuentes) y la práctica efectiva de la enseñanza (Godino et al., 2012). Además, la GALT-proporcionalidad se puede aplicar no solo para el análisis de lecciones de libros de texto, sino para apoyar un análisis sistemático de cualquier proceso de instrucción planificado o implementado sobre el tema de la proporcionalidad. 


\section{Financiamiento}

Trabajo realizado en el marco del proyecto de investigación PID2019-105601GB-I00 / AEI / 10.13039/501100011033 (Ministerio de Ciencia e Innovación). Se reconoce el apoyo de la Universidad de Costa Rica por adjudicación de una beca para estudios en el exterior.

\section{Conflicto de intereses}

Los autores declaran no tener algún conflicto de interés.

\section{Declaración de la contribución de los autores}

Todos los autores afirmamos que se leyó y aprobó la versión final de este artículo. El porcentaje total de contribución para la conceptualización, preparación y corrección de este artículo fue el siguiente: M.C.C. $40 \%$, M.C.C. $30 \%$, J.D.G. $30 \%$.

\section{Declaración de disponibilidad de los datos}

Los datos que respaldan los resultados de este estudio serán puestos a disposición por el autor correspondiente [M.C.C], previa solicitud razonable.

\section{Referencias}

American Association for the Advancement of Science (AAAS) (2000). Middle grades mathematics textbooks: A benchmarks-based evaluation. http://www.project2061.org/publications/textbook/mgmth/report/part1.htm

Aroza, C. J., Godino, J. D. \& Beltrán-Pellicer, P. (2016). Iniciación a la innovación e investigación educativa mediante el análisis de la idoneidad didáctica de una experiencia de enseñanza sobre proporcionalidad. Aires, 6(1), 1-29.
Ávila, A. (2013). La alfabetización matemática y su relación con el intercambio comercial, la escolaridad elemental y el trabajo. Bolema, 27(45), 31-53.

Behr, M., Harel, G., Post, T. \& Lesh, R. (1992). Rational number, ratio and proportion. En D. Grouws (Ed.), Handbook of research on mathematics teaching and learning (pp. 296333). Nueva York, NY: MacMillan.

Beltrán-Pellicer, P. \& Godino, J. (2019). An onto-semiotic approach to the analysis of the affective domain in mathematics education. Cambridge Journal of Education, 50(1), 1-20.

Ben-Chaim, D., Fey, J., Fitzgerald, W., Benedetto, C. \& Miller, J. (1998). Proportional reasoning among 7th grade students with different curricular experiences. Educational Studies in Mathematics, 36(3), 247-273.

Ben-Chaim, D., Keret, Y. \& Ilany, B. S. (2012). Ratio and proportion: Research and teaching in mathematics teachers' education. Róterdam, Países Bajos: Sense Publisher.

Bolea, P., Bosch, M. \& Gascón, J. (2001). La transposición didáctica de organizaciones matemáticas en procesos de algebrización: el caso de la proporcionalidad. Recherches en Didactique des Mathématiques, 21(3), 247-304.

Bosch, M. (1994). La dimensión ostensiva en la actividad matemática. El caso de la proporcionalidad (Tesis doctoral). Universidad Autónoma de Barcelona, España.

Breda, A., Font, V. \& Pino-Fan, L. (2018). Criterios valorativos y normativos en la Didáctica de las Matemáticas: el caso del constructo idoneidad didáctica. Bolema, 32(60), 255-278.

Brousseau, G. (1997). The theory of didactical situations in mathematics. Dordrecht, Países Bajos: Kluwer.

Burgos, M. \& Godino, J. D. (2018). Trabajando juntos situaciones introductorias de razonamiento proporcional en primaria. Análisis de una experiencia de enseñanza centrada en el profesor, en el estudiante y en el contenido. Bolema, 33(63), 389-410.

Butto, C. \& Rojano, T. (2010). Pensamiento algebraico temprano: El papel del entorno Logo. Educación Matemática, 22(3), 55-86.

Carraher, T., Carraher, D. \& Schliemann, A. (1991). En la vida diez, en la escuela cero. México: Siglo XXI Editores. 
Castillo, M. J., Burgos, M. \& Godino, J. D. (2021). Elaboración de una guía de análisis de libros de texto de matemáticas basada en la idoneidad didáctica. Educação e Pesquisa (en prensa).

Cohen, L., Manion, L. \& Morrison, K. (2011). Research methods in education. Londres: Routledge.

Cramer, K. \& Post, T. (1993a). Connecting research to teaching proportional reasoning. Mathematics Teacher, 86(5), 404-407.

Cramer, K. \& Post, T. (1993b). Making connections: A case for proportionality. Arithmetic Teacher, 60(6), 342-346.

Cramer, K., Post, T. \& Currier, S. (1992). Learning and teaching ratio and proportion: Research implications. En D. T. Owens (Ed.), Research ideas for the classroom: Middle grade mathematics (pp. 159-178). Nueva York, NY: MacMillan.

Fan, L., Zhu, Y. \& Miao, Z. (2013). Textbook research in mathematics education: development status and directions. ZDM, 45(5), 633-646.

Fernández, A. (2001). Precursores del racionamiento proporcional: un estudio con alumnos de primaria (Tesis doctoral). Universidad de Valencia, Valencia, España.

Fernández, A. \& Puig, L. (2002). Análisis fenomenológico de los conceptos de razón, proporción y proporcionalidad. La Gaceta de la RSME, 5(2), 397-416.

Fernández, C. \& Llinares, S. (2011). De la estructura aditiva a la multiplicativa: efecto de dos variables en el desarrollo del razonamiento proporcional. Infancia y Aprendizaje, 34(1), 67-80.

Fiol, M. L. \& Fortuny, J. M. (1990). Proporcionalidad directa. La forma y el número. Madrid: Síntesis.

Freudenthal, H. (1983). Didactical phenomenology of mathematical structures. Dordrecht, Países Bajos: Reidel Publishing Company.

Gairín, J. \& Oller, A. (2012). Análisis histórico sobre la enseñanza de la razón y la proporción. En A. Estepa, Á. Contreras, J. Deulofeu, M. C. Penalva, F. J. García y L. Ordóñez (Eds.), Investigación en Educación Matemática XVI (pp. 249-259). Jaén, España: SEIEM.

Godino, J. D. (2013). Indicadores de la idoneidad didáctica de procesos de enseñanza y aprendizaje de las matemáticas. Cuadernos de Investigación y Formación en Educación Matemática, 11, 111-132.
Godino, J. D., Batanero, C. \& Font, V. (2007). The onto-semiotic approach to research in mathematics education. ZDM, 39(1-2), 127-135.

Godino, J. D., Burgos, M., Beltrán-Pellicer, P., Gea, M. \& Giacomone, B. (2019). Structuring the system of didactical suitability criteria of mathematics instruction processes. CIEAEM 71, Braga, Portugal.

Godino, J., Font, V. \& Wilhelmi, M. (2006). Análisis ontosemiótico de la lección sobre la suma y la resta. Revista Latinoamericana de Investigación en Matemática, 9 (especial), 131-155.

Godino, J., Rivas, H. \& Arteaga, P. (2012). Inferencia de indicadores de idoneidad didáctica a partir de orientaciones curriculares. Praxis Educativa, 7(2), 331-354.

Grupo Beta (1990). Proporcionalidad geométrica y semejanza. Madrid, Granada: Síntesis.

Guacaneme, E. (2001). Estudio didáctico de la proporción y la proporcionalidad: Una aproximación a los aspectos matemáticos formales y a los textos escolares de matemáticas (Tesis de maestría no publicada). Universidad del Valle, Cali, Colombia.

Hart, K. (1988). Ratio and proportion. En J. Hiebert \& M. Behr (Eds.), Concepts and operations in the Middle Grades, 2. (pp. 198-219). Reston, VA: National Council of Teachers of Mathematics.

Karplus, R., Pulos, S. \& Stage, E. (1983). Early adolescents proportional reasoning on "rate" problems. Educational Studies in Mathematics, 14(3), 219-233.

Lamon, S. (1993). Ratio and proportion: connecting content and children's thinking. Journal for Research in Mathematics Education, 24(1), 41-61.

Lamon, S. (2006). Teaching fractions and ratios for understanding. Mahwah, Nueva Jersey: Erlbaum.

Lamon, S. (2007). Rational numbers and proportional reasoning: Toward a theoretical framework. En F. Lester (Ed.), Second handbook of research on mathematics teaching and learning (pp. 629-668). Charlotte, Carolina del Norte: Information Age Publishing.

Lesh, R., Post, T. \& Behr, M. (1988). Proportional reasoning. En J. Hiebert and M. Behr (Eds.), Number concepts and operations in the middle grades (pp. 93-118). Reston, Virginia: The National Council of Teachers of Mathematics. 
Lim, K. H. (2009). Burning the candle at just one end: Using non-proportional examples helps students determine when proportional strategies apply. Mathematics Teaching in the Middle School, 14(8), 492- 500.

Martínez, A. (2015). Examining students' proportional reasoning strategy levels as evidence of the impact of an integrated LEGO robotics and mathematics learning experience. Journal of Technology Education, 26(2), 46-73.

Ministerio de Educación, Cultura y Deporte (MECD) de España (2014a). Real Decreto 126/2014, de 28 de febrero, por el que se establece el currículo básico de la Educación Primaria. Madrid: MECD.

Ministerio de Educación, Cultura y Deporte (MECD) de España (2014b). Real Decreto1105/2014, de 26 de diciembre, por el que se establece el currículo básico de la Educación Secundaria Obligatoria y del Bachillerato. Madrid: MECD.

Mochón, S. (2012). Enseñanza del razonamiento proporcional y alternativas para el manejo de la regla de tres. Educación Matemática, 24(1), 133-157.

Modestou, M., Elia, I., Gagatsis, A. \& Spanoudis, G. (2008). Behind the scenes of pseudo-proportionality. International Journal of Mathematical Education in Science and Technology, 39(3), 313-324.

Monterrubio, M. C. \& Ortega, T. (2011). Diseño y aplicación de instrumentos de análisis y valoración de textos escolares de Matemáticas. PNA, 5(3), 105-127.

National Council of Teachers of Mathematics (NCTM) (2000). Principles and Standards for School Mathematics. Reston, Virginia: NCTM.

Obando, G., Vasco, C. \& Arboleda, L. (2014). Enseñanza y aprendizaje de la razón, la proporción y la proporcionalidad: un estado del arte. Revista Latinoamericana de Investigación en Matemática Educativa, 17(1), 59-81.

Piaget (1978). Psicología del niño. Madrid: Morata.

Rivas, M. (2013). Análisis epistémico y cognitivo de tareas de proporcionalidad en la formación de profesores de educación primaria (Tesis doctoral). Universidad de Granada, España.

Ruiz, E. \& Valdemoros, M. (2006). Vínculo entre el pensamiento proporcional cualitativo y cuantitativo: el caso de Paulina. Revista Latinoamericana de Investigación en Matemática Educativa, 9(2), 299-324.
Shield, M. \& Dole, S. (2013). Assessing the potential of mathematics textbooks to promote deep learning. Educational Studies in Mathematics, 82(2), 183-199.

Silvestre, A. \& Da-Ponte, J. (2011). Una experiencia de enseñanza dirigida al desarrollo del razonamiento proporcional. Revista Educación y Pedagogía, 23(59), 137-158.

Soto, I. \& Rouche, N. (1995). Problemas de proporcionalidad resueltos por campesinos chilenos. Educación Matemática, 7(1), 77-95.

Tourniaire, F. \& Pulos, S. (1985). Proportional reasoning: A review of the literature. Educational Studies in Mathematics, 16(2), 181-204.

Van Dooren, W., De Bock, D., Gillard, E. \& Verschaffel, L. (2009). Add? or multiply? A study on the development of primary school students' proportional reasoning skills. En M. Tzekaki, M. Kaldrimidou y C. Sakonidis (Eds.), Proceedings of the 33rd Conference of the International Group for the Psychology of $\mathrm{Ma}$ thematics Education (Vol. 5, pp. 281-288). Thessalonika, Grecia: PME.

Van Dooren, W., De Bock, D., Depaepe, F., Janssens, D. \& Verschaffel, L. (2003). The illusion of linearity: expanding the evidence towards probabilistic reasoning. Educational Studies in Mathematics, 53(2), 113-138.

Vandercruysse, S., Ter Vrugte, J., De Jong, T., Wouters, P., Van Oostendorp, H., Verschaffel, L., Moeyaert, M. \& Elenet, J. (2016). The effectiveness of a math game: the impact of integrating conceptual clarification as support. Computers in Human Behavior, 64, 21-33.

Vergnaud, G. (1983). Multiplicative structures. En R. Lesh y M. Landau (Eds.), Acquisition of mathematical concepts and processes (pp. 127-174). Orlando, Florida: Academic.

Wilhelmi, M. R. (2017). Proporcionalidad en educación primaria y secundaria. En J. M. Contreras, P. Arteaga, G. R. Cañadas, M. M. Gea, B. Giacomone y M. M. López-Martín (Eds.), Actas del Segundo Congreso International Virtual sobre el Enfoque Ontosemiótico del Conocimiento y la Instrucción Matemáticos. http://enfoqueontosemiotico.ugr.es/civeos/ wilhelmi.pdf 


\section{(c) (1) (ㄱ) $\Theta$}

Guía de análisis de lecciones de libros de texto de Matemáticas en el tema de proporcionalidad (María José Castillo Céspedes • María Burgos Navarro • Juan Díaz Godino) Uniciencia is protected by Attribution-NonCommercial-NoDerivs 3.0 Unported (CC BY-NC-ND 3.0) 